OPEN ACCESS

Edited by:

Martin G. Klotz,

Washington State University,

United States

Reviewed by:

Manuel Serif,

Norwegian University of Science

and Technology, Norway

Peter Hegemann Peter,

Humboldt University of Berlin,

Germany

Zahir Ali,

King Abdullah University of Science and Technology, Saudi Arabia

*Correspondence:

Mark Andrew Moosburner

mmoosbur@ucsd.edu Andrew E. Allen aallen@ucsd.edu

Specialty section: This article was submitted to

Aquatic Microbiology,

a section of the journa

Frontiers in Microbiology

Received: 09 April 2019 Accepted: 03 January 2020

Published: 28 January 2020

Citation:

Moosburner MA, Gholami P, McCarthy JK, Tan M, Bielinski VA and

Allen AE (2020) Multiplexed Knockouts in the Model Diatom Phaeodactylum by Episomal Delivery of a Selectable Cas9. Front. Microbiol. 11:5. doi: 10.3389/fmicb.2020.00005

\section{Multiplexed Knockouts in the Model Diatom Phaeodactylum by Episomal Delivery of a Selectable Cas9}

\author{
Mark Andrew Moosburner1,2*, Pardis Gholami2, James K. McCarthy'2, Maxine Tan 1,2, \\ Vincent A. Bielinski ${ }^{2}$ and Andrew E. Allen ${ }^{1,2 *}$ \\ 1 Integrative Oceanography Division, Scripps Institution of Oceanography, University of California, San Diego, La Jolla, CA, \\ United States, ${ }^{2}$ J. Craig Venter Institute, La Jolla, CA, United States
}

Marine diatoms are eukaryotic microalgae that play significant ecological and biogeochemical roles in oceans. They also have significant potential as organismal platforms for exploitation to address biotechnological and industrial goals. In order to address both modes of research, sophisticated molecular and genetic tools are required. We presented here new and improved methodologies for introducing CRISPRCas9 to the model diatom Phaeodactylum tricornutum cells and a streamlined protocol for genotyping mutant cell lines with previously unknown phenotypes. First, bacterialconjugation was optimized for the delivery of Cas9 by transcriptionally fusing Cas9 to a selectable marker by the $2 \mathrm{~A}$ peptide. An episome cloning strategy using both negative and positive selection was developed to streamline CRISPR-episome assembly. Next, cell line picking and genotyping strategies, that utilize manual sequencing curation, TIDE sequencing analysis, and a T7 endonuclease assay, were developed to shorten the time required to generate mutants. Following this new experimental pipeline, both singlegene and two-gene knockout cell lines were generated at mutagenesis efficiencies of $48 \%$ and $25 \%$, respectively. Lastly, a protocol for precise gene insertions via CRISPRCas9 targeting was developed using particle-bombardment transformation methods. Overall, the novel Cas9 episome design and improved genotyping methods presented here allow for quick and easy genotyping and isolation of Phaeodactylum mutant cell lines (less than 3 weeks) without relying on a known phenotype to screen for mutants.

Keywords: diatom, gene editing, CRISPR-Cas9, conjugation, multiplex, bioengineering, mutant, Phaeodactylum

\section{INTRODUCTION}

Marine diatoms are of biotechnological significance for their capacity to assimilate large amounts of carbon and nitrogen, divide rapidly (Cermeño et al., 2011), thrive at high cell densities, and fix carbon via photosynthesis. Diatoms shunt nutrients toward metabolic processes that produce industrially valuable products such as biodiesel precursors (triglycerides a.k.a. TAGs), polyunsaturated fatty acids (PUFAs), and photosynthetic pigments (Pulz and Gross, 2004; Vinayak et al., 2015). Regardless of their innate ability to produce these high-valued molecules (HVMs),

Abbreviations: GA, Gibson assembly; GG, golden gate assembly; Phaeodactylum, Phaeodactylum tricornutum; RT, room temperature. 
increasing production beyond their native capabilities is desirable for reducing production costs, and achieving sustainability goals in industrial agriculture applications (Gao, 2018). Utilization of genetic engineering technologies could shift metabolic outputs of marine diatoms toward these HVMs and improve commercial production where algae have been suggested as biological platforms for industrial feedstock and nutraceutical production (Vinayak et al., 2015). Nevertheless, the development and optimization of such genetic engineering technologies is necessary before they can be safely, easily, and robustly employed.

Genetic engineering technologies, particularly the versatile CRISPR-Cas9 toolbox, have been used for numerous applications in a vast array of organisms (Mali et al., 2013b; Sanders and Joung, 2014; Wang et al., 2016). CRISPR (clustered regularly interspaced short palindromic repeats), a foreign nucleic acid immunity system, is encoded in the genomes of most bacteria and some archaeal species (Deltcheva et al., 2011). The typeII CRISPR system from the bacterium Streptococcus pyogenes was first adapted for gene editing using the Cas9 enzyme, which induces blunt-ended double-stranded breaks (DSBs) when guided to a genetic target by a chimeric RNA molecule of the tracr-RNA and cr-RNA called a single-guided RNA (sgRNA) (Cong et al., 2013; Jinek et al., 2013; Mali et al., 2013a). Together Cas9 and an sgRNA form a ribo-endonuclease complex in vivo in many cell types to edit or mutate genomic targets. In the bacterial immunity system, the sgRNA targeting sequence, a string of $\sim 20$ nucleotides ( $\mathrm{nt}$ ), preserves the sequence of a previous immune response, called spacer acquisition (McGinn and Marraffini, 2018); whereas in gene editing applications the nucleotide sequence of 20-nt spacer is designed by the researcher to target a specific gene/target, followed by interrogation and cleavage of a nucleic acid target by the Cas 9 endonuclease. The sgRNA guides Cas 9 to the target by forming complementary base pairing with the $20 \mathrm{nt}$ at the $5^{\prime}$ end of the sgRNA, called the spacer. Once a DSB is induced, the blunt ended breaks are repaired by one of two native DNA repair mechanisms: homology-directed repair (HDR) or non-homologous end joining (NHEJ). HDR relies on a homologous donor to repair the DSB, typically a sister chromosome in eukaryotes, by homologous recombination (LaFave and Sekelsky, 2009). Endogenous HDR can be hijacked in order to introduce, at the DSB, an exogenous donor sequence, containing a user-designed mutation that will result in an edited target locus. For NHEJ, ligation of the blunt ends repairs the DSB, however, this process is error-prone and leads to random insertions or deletions of nucleotides of unpredictable sizes called indels (Davis and Chen, 2013).

CRISPR-Cas9 has been employed in both prokaryotic and eukaryotic organisms but eukaryotes pose particular challenges for CRISPR-Cas9 experiments as most have multiple copies of their chromosomes. Multiple copies of chromosomes provide two or more target loci for Cas9. For instance, in a diploid organism, two individual DNA repair events must occur to produce homozygous or heterozygous mutations at the target loci. For NHEJ-mediated mutagenesis (where no homologous donor is introduced), a heterozygous genotype arises after two separate Cas9 cleavage events and NHEJ-mediated repair that results in two distinct indels at the target loci. A homozygous genotype can also arise when one indel-containing target locus is used as a homologous donor for the second target loci (located on the sister chromosome of the first target), thereby producing an identical indel mutation. The unpredictability of NHEJmediated mutagenesis in diploid organisms therefore requires highly sensitive genotyping methodologies. Many genotyping methods have been used to genotype diploid organisms; among the most common are high-resolution melt curve analysis (HRMC) (Nymark et al., 2016), T7 endonuclease I assay (Guschin et al., 2010; Cho et al., 2013), high-throughput next generation sequencing (Zhou et al., 2014), and manual curation of sequencing reads.

Marine diatom species have been subjected to CRISPRCas9 mutagenesis including the model strains: the pennate, Phaeodactylum tricornutum (Nymark et al., 2016; Serif et al., 2018; Sharma et al., 2018; Slattery et al., 2018; Stukenberg et al., 2018) and the centric Thalassiosira pseudonana (Hopes et al., 2016). To date, NHEJ-mediated mutagenesis has been observed only in Phaeodactylum (Nymark et al., 2016; Serif et al., 2018; Slattery et al., 2018) by using one of two genetic transformation methods, micro-particle bombardment or bacterial conjugation, to introduce CRISPR-Cas9. Despite the successes of utilizing CRISPR-Cas9 in generating mutant diatom cell lines, genotyping the generated cell lines has been difficult. Phaeodactylum, a diploid organism, requires highsensitivity screening methods to genotype multiple cell lines in a high-throughput manner. Genotyping methods such as high-throughput melt curve (HRMC) analysis and the T7 endonuclease I assay are commonly used to evaluate mutant genotypes in multi-ploidy organisms. To date, HRMC analysis and CRISPR Activity Factor (CAF) analysis have been used for Phaeodactylum genotyping experiments (Nymark et al., 2016; Sharma et al., 2018; Stukenberg et al., 2018). Similarly to CAF, TIDE (Tracking Indels by DEconvolution) sequencing analysis, an open-source software package, deconvolves raw sequencing reads to reveal indels in diploid or multiploidy organisms (Brinkman et al., 2014; Serif et al., 2018). Allele-specific PCR amplification has been used for genotyping Phaeodactylum mutants without the need for subcloning (Serif et al., 2018).

Here, TIDE sequencing analysis and manual sequencing curation is used to streamline the genotyping pipeline from colony formation to mutant cell line validation. Both singlegene and two-gene mutant cell lines were produced utilizing conjugation to introduce a selectable-Cas9 and either one or two sgRNAs to Phaedocatylum. Also, the T7 endonuclease I assay has also been adapted to quantify the mutagenesis efficiency of individual sgRNAs throughout a population of transformed Phaeodactylum prior to genotyping individual colonies.

A new selectable-Cas9 episome design was used that permits antibiotic selection for the Cas9 gene with the goal of both increasing mutagenesis efficiency and potentially avoiding mixed genotypes forming in generated colonies after conjugation. The P2A self-cleaving peptide was used to transcriptionally fuse Cas9 and the Phaeodactylum antibiotic resistant gene, sh ble (Kim et al., 2011). By doing so, Cas 9 and sh ble are co-transcribed under the same promoter followed by cleavage of the translated products, essentially selecting for Cas9 via antibiotic treatment. Lastly, a 
robust golden-gate assembly based method was developed to visualized positive and negative $E$. Coli clones that harbor readyto-conjugate episomes. Overall, the methods presented here allow for easy Cas9-sgRNA episome assembly and streamlined genotyping methodology and isolation of Phaeodactylum mutant cell lines in less than 3 weeks without relying on a known phenotype to screen for mutants.

\section{MATERIALS AND METHODS}

\section{Cell Culturing}

A reference strain of Phaeodactylum (CCAP-1055/1) was used in all experiments. Phaeodactylum was grown at $18^{\circ} \mathrm{C}$ under white fluorescent lights $\left(50 \mathrm{uE} \mathrm{m}^{-2} \mathrm{~s}^{-1}\right)$ and subjected to a diel growth cycle (14 h:10 h; light:dark). Culture medium was artificial sea water (ASW) supplemented with trace metals, essential vitamins, $55 \mathrm{uM}$ of $\mathrm{NaPO}_{4}$, and $880 \mathrm{uM}$ of the appropriate nitrogen source $\left(\mathrm{NaNO}_{3}, \mathrm{NH}_{4} \mathrm{Cl}\right.$, or Urea). Cultures, when not used in growth assays, were maintained with chloramphenicol antibiotic $(10 \mathrm{mg} / \mathrm{L})$ to keep cultures bacteria-free. Mutant Phaeodactylum strains were supplemented with either phleomycin $(50 \mathrm{mg} / \mathrm{L})$, zeocin $(50 \mathrm{mg} / \mathrm{L})$, or nourseothricin $(200 \mathrm{mg} / \mathrm{L})$.

Escherichia coli (TOP-10, Life Technologies, Carlsbad, CA) was used for all molecular cloning purposes. The cultures were grown on Luria-Bertani broth or agar and supplemented with the following antibiotics when necessary: ampicillin $(100 \mathrm{mg} / \mathrm{L})$, carbicillin (100 $\mathrm{mg} / \mathrm{L})$, tetracyclin $(10 \mathrm{mg} / \mathrm{L})$, gentamicin (20 mg/L), zeocin (25 mg/L).

\section{Molecular Biology}

Plasmid construction was performed with reference to Sambrook and Russell (2001) unless stated otherwise. Multiple DNA polymerases were used for cloning purposes including Phusion High-Fidelity DNA polymerase (Thermo Fisher Scientific, Waltham, MA, United States), AccuPrime Taq High-Fidelity DNA polymerase (Thermo Fisher Scientific), OneTaq 2X Master Mix with standard buffer (New England Biolabs, Ipswich, MA, United States), and Phire Plant Direct PCR Master Mix (Thermo Fisher Scientific). Enzymatic components to make a 2X GA master mix that included Phusion HF DNA polymerase, T5 exonuclease (Thermo Fisher Scientific), and DNA Taq Ligase (Thermo Fisher Scientific) were purchased separately and mixed in lab. Enzymatic components for Golden-Gate cloning were also purchased separately but only mixed on the day of cloning. They included the type-II restriction enzymes BsaI-HF and BbsI (New England Biolabs) and a T4 DNA Ligase (Thermo Fisher Scientific).

Primer sequences can be found in the Supplementary Material.

\section{CRISPR/Cas9 Genetic Components}

A human-codon optimized Cas9 open reading frame (ORF) containing three nucleus-localization signals (NLS) was produced by the Feng Zhang laboratory (Broad Institute, Cambridge, MA, United States) and purchased from addgene.com [pSpCas9(BB)-2A-Puro (PX459) V2.0, ID:
62988, Ran et al., 2013]. The Cas9 ORF was cloned into a pUC19 backbone using GA cloning (Gibson et al., 2009) using primers Cas9-2A-shble_1 and P2A_1 (Supplementary Material). For constitutive expression of Cas9, the FcpB/FcpA (Fucoxanthin chlorophyll a/c promoter B/Fucoxanthin chlorophyll a/c terminator A) (Bailleul et al., 2010) promoter/terminator pair were chosen (FcpB-Cas9).

The HDR-donor plasmid used in Weyman et al. (2014) for TALEN mutagenesis was adapted to a HDR-donor plasmid for targeting nitrate reductase ( $\mathrm{pKO}-\mathrm{NR}$ ). $\mathrm{pKO}-\mathrm{NR}$ contained $1 \mathrm{~kb}$ homology arms flanking an expression cassette coding for the $s h$ ble gene that conferred resistance to the antibiotic phleomycin. The cassette inserted both a sh ble cassette and a STOP codon upstream of the sh ble promoter (Fucoxanthin chlorophyll a/c binding promoter $\mathrm{F}, \mathrm{FcpF}$ ). This was in frame with the targeted coding gene. The primers and plasmid sequence information can be found in Weyman et al. (2014).

The sRNAi promoter (chr2: 28,038-29,083) and terminator (chr2: 29,124-29,424) was amplified from Phaeodactylum genomic DNA (promoter primers = sRNAi1_1 and sRNAi1_2; terminator primers $=$ sRNAi_3 and sRNAi_4). For Golden Gate cloning, the sRNAi terminator was shortened by PCR to $112 \mathrm{bp}$ to eliminate an internal $B s a \mathrm{I}$ restriction site. The sRNAi promoter and terminator was cloned from Phaeodactylum genomic DNA at the locus of the most highly expressed small, non-coding, intergenic RNA called sRNAi (Rogato et al., 2014; Bielinski et al., 2017). The sequences for the sRNAi promoter and terminator can be found in the Supplementary Material.

The sgRNA backbone, which consists of the genetic fusion of the crRNA and tracrRNA (Jinek et al., 2012), was amplified from the same Addgene plasmid that contained Cas9 (primers $=$ sRNAgU1_F and sRNAgU1_R). An sgRNA expression cassette for Phaeodactylum (sRNAi_gBbsI) was constructed using a 4-piece GA approach into an XbaI-digested pUC19 plasmid containing a bacterial expression cassette for the bla gene conferring resistance to ampicillin/carbicillin. The resulting plasmid was then PCR amplified at the $3^{\prime}$ end of the sRNAi and the $5^{\prime}$ end of the sgRNA backbone (primers = gRNA_1 and gRNA_2). This amplicon served as a linearized vector for a 2-piece GA procedure for a spacer insert that contains two flanking BbsI restriction sites (Supplementary Figure S2). The 100 bp GA insert was constructed by ligating two 60 bp oligos with homology to the sRNAi promoter and sgRNA backbone. The resulting plasmid, $s R N A i$ g2XBbsI, was used for BbsI-based GG cloning of CRISPR spacers (Supplementary Figure S2B).

For all Cas9 target genes, spacer sequences were obtained for nitrate reductase (Phatr3_J54983), glutamine synthetase II (GS-2) (Gene ID: Phatr3_J51092), and chloroplastic glutamate synthase (cGOGAT) (Gene ID: Phatr3_J24739) using the Phyto CRISPEx online package (Rastogi et al., 2016) that passed the built-in criteria of minimizing off-target Cas9 activity.

A GG cloning protocol was modified from the sgRNA expression cassette supplied by Feng Zhang laboratory. In place of the BbsI-containing spacer sequence within the sgRNA expression plasmid, a LacZ bacterial expression cassette with flanking BbsI sites was cloned (primers = LacZ_GG_1 and LacZ_GG_2) into the sRNAi expression cassette using GA. The Pt 
sgRNA expression plasmid previously produced (sRNAi_gBbsI) was PCR amplified (primers = LacZ_GG_3 and LacZ_GG_4) and the amplicon was gel extracted and used as a GA vector. The sgRNA cloning vector (sRNAi_gLacZ) was the assembled plasmid. This plasmid was used in all future sgRNA expression cassette cloning and assembly experiments as a cloning vector (Supplementary Figure S1B). An appropriate GG insert for sRNAi_gLacZ was assembled by ligating two 56-nt oligos together that are complimentary to each other. The oligo primer sequences for all sgRNA target sequences can be found in Supplementary Material.

Detailed protocols for spacer cloning into the sRNAi_gLacZ plasmid (name GG1 cloning) are available at protocols.io (doi: 10.17504/protocols.io.4abgsan).

\section{Cas9-Episomal Plasmid Cloning}

The Phaeodactylum episomal plasmid pPtPBR1 was obtained from R. Diner (Diner et al., 2016). First, one BsaI restriction site within the bla coding region of pPtPBR1 was removed by PCR amplification (primers $=$ pBR322-Amp_1 and pBR322Amp_2) and reassembly to produce plasmid pPtPBR1 (BsaI-). pPtPBR1 was then used as a template for GA cloning of a Cas9 ORF and a GG cloning BsaI acceptor site for sgRNA expression cassette cloning (Supplementary Figure S2). Cas9 was PCR amplified twice using one forward primer (Cas9-2Ash ble_1) and two reverse primers (P2A_1 and P2A_2) so to add GA homology to the FcpF promoter ( $5^{\prime}$ of Cas9) and the sh ble ORF ( $3^{\prime}$ of Cas9). The double PCR reaction added a GSG-2A peptide DNA coding sequence to transcriptionally fuse hCas9 and sh ble (Supplementary Figure S2, GSG-P2A nucleotide sequence is in Supplementary Material). Next, a red-fluorescent protein bacterial expression cassette (mRFP) was obtained and PCR amplified (primers = Cas9-2A_sh bleRFP_1 and 2) to produce GG acceptor sites. The remaining two GA pieces were PCR amplified from pPtPBR1 (BsaI-) to insert two GG cloning BsaI sites between the bla and tet expression cassettes and flanking mRFP, one using primers Cas9-2A-sh ble_2 and $3\left(5^{\prime}\right.$ - sh ble - Pt_Centromere pMB1 - bla $-3^{\prime}$ ) and two using primer Cas9-2A-sh ble_6 and $7\left(5^{\prime}\right.$ - tet - OriT - FcpF - $\left.3^{\prime}\right)$. After cloning and E. coli transformation, the correctly assembled episome, pBR_Cas9-2Ash ble, 2XBsaI-mRFP(AT) (Supplementary Figure S3A), was sequence verified.

Next, primers were designed to PCR amplify up to six sgRNA expression cassettes (cloning and construction described above) and to add BsaI-restriction and cleavage sites to the $5^{\prime}$ and $3^{\prime}$ ends of the sRNAi promoter/terminator. GG cloning is an optimal assembly platform when cloning more than one sgRNA expression cassette. For inserting one sRNAi_sgRNA cassette, primers GG-gRNA1-F and sgRNA(GG)-2 were used. For two sgRNAs, primers GG-gRNA1-F and R were used for sgRNA \#1 and primers GG-gRNA3-F and sgRNA(GG)-2 were used for sgRNA \#2. Between the two sgRNAs was cloned a LacZ expression cassette, which, when induced with IPTG activates beta-galactidose that digests Xgal to produce blue E. coli colonies (Supplementary Figure S3C). The sgRNA expression cassette(s) and LacZ amplicons was mixed at double the molar concentration of the BsaI-digested pBR_Cas9-2A-sh ble, 2XBsaI$\mathrm{mRFP}(\mathrm{AT})$ vector and carried through the GG cloning reaction with BsaI-HF and a T4-ligase (Supplementary Figure S1C). The final sgRNA-LacZ-sgRNA episomal plasmid map can be seen in Supplementary Figure S3B.

Detailed protocols for sgRNA cloning into the Cas9-2A-sh ble epsiome (named GG2 cloning) is available at protocols.io (doi: 10.17504/protocols.io.4acgsaw).

\section{Phaeodactylum tricornutum Genetic Transformations \\ Bacterial-Mediated Conjugation}

Bacterial-mediated conjugation was used to introduce CRISPR components to Phaeodactylum. Diner et al., 2016 provided detailed methodology for introducing episomal plasmids to Phaeodactylum in a high-throughput manner. For all transformations, $100 \mathrm{uL}$ of dense Phaeodactylum (1e8 cells $/ \mathrm{mL}$ ) was plated on conjugation-based solid agar medium (NH4/NO3ASW, $1 \%$ agar, 5\% LB in $\mathrm{NH}_{4}$-supplemented ASW) in 6-well cell culture plates and incubated for 4 days under light (18:6) and at $18^{\circ} \mathrm{C}$. Prior to transformation, pBR_Cas9-2A-sh ble, sRNAi-sgRNA episomes were transformed into recipient E. coli harboring the conjugation plasmid pTA-MOB (Karas et al., 2015; Diner et al., 2016) and selected on agar plates for both the cargo and conjugation plasmids (Figure 1). For a transformation control (positive for colonies not Cas9 cleavage), the episome pBR_Cas9-2A-sh ble, 2XBsaI_gRFP1 was used where Cas9 expression would still occur without an accompanying sgRNA. For a negative control, a similar episomal plasmid was built harboring the NAT gene that confers resistance to nourseothricin and not phleomycin. Under phleomycin selection, the transformed cells would die. Transformation efficiency for the delivery of Cas9-2A-sh ble:sgRNA episomes was 500-750 transformants per $1 \times 10^{8}$ phleomycin-resistant cells.

The day before transformation, 4 random colonies from the selection plates were used to inoculate $3 \mathrm{~mL}$ of LB containing selection (Amp, Tet, Gent). After growing overnight, $200 \mathrm{uL}$ of culture was used to inoculate $12.5 \mathrm{~mL}$ of LB. The culture was grown for 3-4 h, until an OD-600 $\mathrm{nm}$ of $0.8-1$ was reached, pelleted, and suspended in 100uL of SOC media. Then, the E. coli was pipetted into a corresponding well containing Phaeodactylum and mixed either by a spreading loop or by rotating the 6-well plate such that the E. coli covered the entire lawn of Phaeodactylum. After E. coli was added to all wells and allowed to dry under a vacuum PCR hood, the 6-well plate was placed in the dark at $30^{\circ} \mathrm{C}$ for $90 \mathrm{~min}$. The plate was then placed back at $18^{\circ} \mathrm{C}$ and exposed to light for $48 \mathrm{~h}$.

Finally, each well was scraped using a sterile cell spreader, collected in a $2 \mathrm{~mL}$ Epitube then re-plated on $100 \mathrm{~mm}$ selection plates containing the appropriate selective media. The selection plates were then allowed to grow for 10-14 days or until colonies appeared. For the T7 Endonuclease I Assay reaction, half of the scraped Phaeodactylum cell lines $(\sim 400 \mathrm{uL})$ was resuspended in $200 \mathrm{~mL} \mathrm{NH}_{4}$-supplemented ASW media supplemented with phleomycin and chloramphenicol. The cells were then grown under light and at $18^{\circ} \mathrm{C}$ for 4 days, passed into $200 \mathrm{~mL}$ fresh 

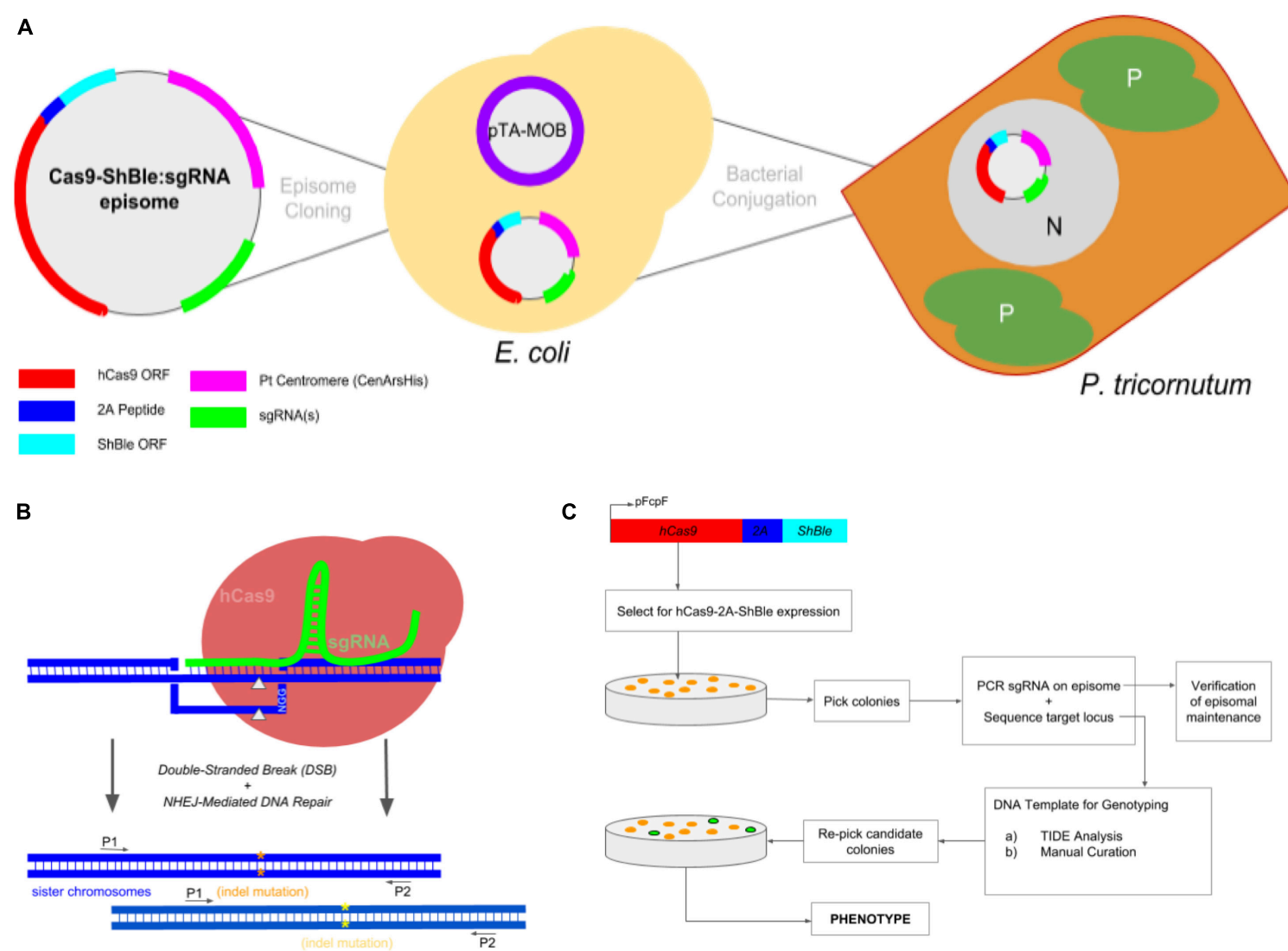

FIGURE 1 | Experimental workflow to produce Phaeodactylum mutants via conjugation-based delivery of a CRISPR-Cas9 episome. (A) A cartoon schematic of Cas9-Sh ble:sgRNA episome cloning to E. coli cells harboring the PTA-MOB plasmid and followed by bacterial-conjugation transformation of the episome to Phaeodactylum. Phaeodactylum cells maintain and replicate the Cas9-Sh ble:sgRNA episome as their native chromosomes in the nucleus (N). The diatom plastids (P) and cytoplasm (C) are labeled. Here, Cas9 is transcriptionally fused to sh ble and can therefore be selected for after transformation with the antibiotic phleomycin. (B) Episomal expression of Cas9 and sgRNA(s) and target mutagenesis of a diploid organism. The sgRNA(s) guides Cas9 to genomic target(s), and then Cas9 induces a double-stranded break (gray arrows). Phaeodactylum, being a diploid eukaryote, will contain two distinct NHEJ-mediated mutations (orange and yellow asterisks) between the sister chromosomes. (C) Genotyping and cell line picking workflow after colony selection for Cas9-Sh ble. A "double picking" strategy was used by which cell lines were picked and target locus sequenced using two primers. After genotyping, the colony can be re-picked for further cell line analysis.

media, grown for an additional 3 days, then finally pelleted and flash frozen.

Detailed protocols for bacterial-conjugation in Phaeodactylum are publicly available at protocols.io (doi: 10.17504/protocols.io. 5 pvg5n6).

\section{Micro-Particle Bombardment Genetic Transformation}

Micro-particle bombardment genetic transformation (Falciatore et al., 1999) was used to introduce CRISPR components to Phaeodactylum. First, Phaeodactylum was pelleted during exponential growth in liquid culture $\left(\mathrm{NO}_{3}\right.$-supplemented ASW), suspended, and plated onto agar growth plates at a cell concentration of $3 \mathrm{e} 8 \mathrm{cells} / \mathrm{mL}(400 \mathrm{uL})$. Next, $24 \mathrm{ug}$ of DNA (8 ug each plasmid; FcpB-Cas9, sRNAi-gNR-B, pKONR) were hybridized to tungsten beads and introduced to the plated Phaeodactylum at high velocity using the PDS-1000/He Biolistic $^{\circledR}$ Particle Delivery System (Bio-Rad, Hercules, CA). The transformed Phaeodactylum plates were then allowed to recover for $48 \mathrm{~h}$ at $18^{\circ} \mathrm{C}$ and in constant darkness. After recovery, Phaeodactylum was re-plated on agar plates containing $\mathrm{NH}_{4}$-supplemented ASW with phleomycin to select for the presence of the pKO-NR plasmid. The selection plates were then grown under normal conditions for 21-28 day, or until Phaeodactylum colonies formed and were visible. Experimental controls were also performed where either FcpB-Cas9 and pKONR were co-transformed or $\mathrm{pKO}$-NR was transformed alone.

\section{T7 Endonuclease I Assay for Determining Cas9 Mutagenesis Efficiency}

The T7 endonuclease I Assay (New England Biolabs) was used to quantify the in vivo cleavage efficiency for multiple clonal Phaeodactylum populations for each sgRNA target. Frozen cell pellets obtained following liquid selection (as described above) were slowly thawed on ice. Genomic DNA was then extracted from the pelleted cells using the Plant DNAzol Reagent (Thermo Fisher Scientific). The product-supplemented protocol was used; however, the pulverization step, the first step, was skipped because diatom pellets can be easily resuspended. Genomic DNA 
was then extracted from the cell pellet. $200 \mathrm{uL}$ of genomic DNA at concentration ranging from 200 to $500 \mathrm{ng} / \mathrm{uL}$ was recovered. A total of $200 \mathrm{ng}$ of genomic DNA was used as a PCR template to start the T7 endonuclease I assay. Six separate amplifications were performed (only 25X amplification cycles) and pooled prior to the T7 assay. For the NR locus, primers NR-gene- 1 and NR-KO-2* were used. This amplification product was then re-amplified with nested primers NR-HRM-A and NR-HRM-B. The nested-amplicons were then PCR cleaned and concentrated and used as a DNA input for the heteroduplex hybridization. This input is referred to as "Amplicon" in Figure 2 and the hybridized heteroduplex is referred to as "Heteroduplex." T7 endonuclease I was then added to the hybridized product to cleave all heteroduplex DNA. All reaction conditions and efficiency calculations (as shown in Figure 2) were supplied by New England Biolabs (protocol website ${ }^{1}$ ). Genome editing efficiency was determined using the Agilent 4200 TapeStation bioanalyzer and Agilent High-Sensitivity D1000 screen chip and assay. The fraction cleaved value was determined by examining the peak intensities of the high-sensitivity gel of the T7 product compared to the uncut Heteroduplex product. Fraction cleaved was then an input for the following equation to calculate the percentage of gene modification:

$$
\% \text { gene modification }=100 *\left(1-(1-\text { fraction cleaved })^{1 / 2}\right) .
$$

\section{Genotyping Phaeodactylum Exconjugants}

Phaeodactylum cells selected on solid media were grown for 1014 day or until visible colonies formed. Colonies were picked with pipette tips directly into $5 \mathrm{uL}$ dilution buffer supplied with the Direct Plant PCR Kit (Thermo Fisher Scientific) and incubated at RT for $15 \mathrm{~min}$. The buffered colony was then pipette mixed and $2 \mathrm{uL}$ were used as a DNA template for two PCR reactions. The first, common for all sgRNAs, used primers Ars_Seq and V2_insert_2 (Supplementary Figure S4A, black arrows) to amplify from the Pt_Centromere to and through the sgRNA expression cassette to check for correct episomal maintenance and sgRNA presence (Supplementary Figure S4B). For nitrate reductase, primers NR-gene-1 and NR-KO-2* were used to amplify a $641 \mathrm{bp}$ region around the target loci for gNRA and gNR-B. The Direct Plant PCR Kit was used for both PCR reactions for 40 cycles. The resulting PCR amplicons were used for genotyping.

\section{TIDE Sequencing Analysis}

TIDE (Tracking of Indels by DEconvolution, Brinkman et al., 2014) sequence analysis is an online-based software ${ }^{2}$ that analyzes raw sequencing data to find one or multiple indels mutations at a Cas9 target site. For the purposes of this study, TIDE was used to analyze indels resulting from Cas9 targeting of nitrate reductase in Phaeodactylum by sgRNAs gNR-A and gNR$\mathrm{B}$ (Figure 3). An example of the TIDE analysis output for a

\footnotetext{
${ }^{1}$ https://www.neb.com/protocols/2014/08/11/determining-genome-targetingefficiency-using-t7-endonuclease-i

${ }^{2}$ https://tide.deskgen.com/
}

wild-type sequence is shown in Figure 3A. 80 colonies for gNR$A$ and 64 colonies for gNR-B were picked directly into PCR mix (protocol described above). 200 ng of the PCR amplicons were then directly sequenced using two primers, NR-HRM-A and NR-gene-2, for paired-end reads (Figure 2A). Phaeodactylum transformed with the Cas9-2A-sh ble vector without an sgRNA was used as a control. TIDE analysis requires the input of (1) the nuclease species used (SpCas9), (2) the 20 nt spacer target, (3) an ABI (.abi) file for a reference sequence (control Cas92A-sh ble), and (4) an ABI (.abi) file for a sample sequence. The sample sequence is compared to the control sequence for significant differences between the sequence electropherograms. Certain criteria were identified and selected for when analyzing the TIDE output. First, a total efficiency value (in percentage) of $50 \%$ was chosen because it signified that the target locus was mutated at a high rate. Second, the $p$-value of each output peak must be $<0.001$. Third, only colonies with distinct genotypes, based on the criteria of only 1 or 2 predominant indels each with greater than $10 \%$ of the total sequencing reads, were selected as candidate mutant lines. Lastly, for the cell lines that passed each of these criteria, results were verified by conducting TIDE analysis of the paired-end read of the same sample. If both paired-end TIDE analyses pass the criteria and identified identical indels, the sample was chosen as a mutant cell line candidate. Each sequencing read was manually analyzed, individually, using the open-sourced TIDE web-tool.

\section{Nitrate Phenotype Assay for Phaeodactylum Mutant Cell Line Candidates}

NR-KO Phaeodactylum cell lines were identified by TIDE and subjected to the NR phenotype assay where Phaeodactylum mutants of nitrate reductase are grown on media supplemented with nitrate and ammonium or urea, separately. The same NR assay was used for mutant cell lines produced via micro-particle bombardment. Mutants with a dysfunctional NR gene are unable to grow on nitrate media while proliferating on ammonium. First, all 40 colonies (for each sgRNA) were re-picked into liquid media supplemented with ammonium. The cultures (including four control cultures) were grown under normal conditions until midlog phase and then passed into nitrate and ammonium media at a concentration of $1 \mathrm{e} 4$ cells $/ \mathrm{mL}$. Cell density was measured daily (2:00pm PST) for 4 days and growth between the two condition was compared. The cell lines that could grow on ammoniumsupplemented ASW and could not grow on nitrate-supplemented ASW were considered NR mutant cell lines.

\section{RESULTS}

\section{CRISPR-Cas9 Mutagenesis Workflow in Phaeodactylum Using Selectable-Cas9 Episome}

The general workflow from episomal exchange to Phaeodactylum colony genotyping is shown in Figure 1. The Phaeodactylum episome was built with a transcriptionally fused Cas9 and sh 


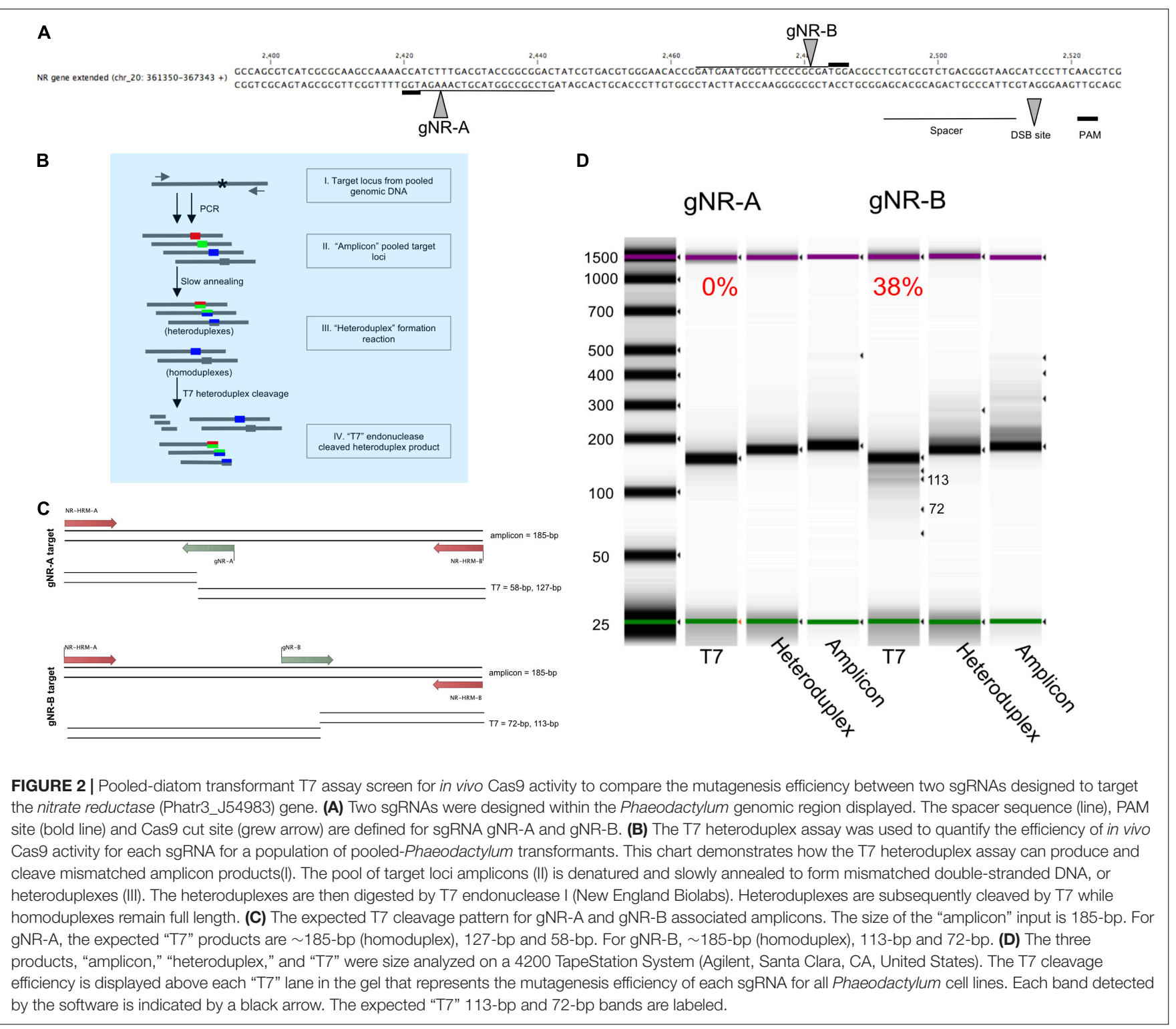

ble, an antibiotic selectable marker gene, expression cassette that allows Cas9 to be selected for after transformation with the eukaryote antibiotic phleomycin. Additionally, a red-fluorescent protein bacterial expression cassette (mRFP) was cloned into the episome with flanking $B s a I$ restriction enzyme sites that, when digested, leave unique 4-bp overhangs for golden-gate cloning of sgRNA constructs (Supplementary Figure S1). A hierarchical cloning strategy was used to build and clone one Phaeodactylum sgRNA expression cassette in two golden-gate assembly steps (Supplementary Figure S2). Negative selection against RFP was used for cloning an individual sgRNA expression cassette where negative $E$. coli colonies were visibly red. For cloning more than one sgRNA, a LacZ bacterial expression cassette was cloned with and between the sgRNAs that turns E. coli colonies with positively assembled episomes blue in the presence of IPTG and Xgal. The red-white-blue screening method helped expedite episome cloning in general (Supplementary Figure S3).
After construction and sequence verification, a Cas9-sh ble:sgRNA episome was transformed into electro-competent E. coli cells harboring a pta-MOB plasmid (Karas et al., 2015; Diner et al., 2016) that has been shown to be required for efficient conjugation exchange of the episome to Phaeodactylum (Figure 1A). The episome then resides in the Phaeodactylum nucleus, separate from the Phaeodactylum chromosomes as an artificial chromosome, where expression of Cas9 and sgRNA(s) occurs. Like many CRISPR/Cas9 gene targeting experiments in organisms with diploid genomes, the Cas9-sgRNA ribo-endonuclease complex is guided to both gene alleles where distinct NHEJ-mediated mutation may occur (Figure 1B). While a heterozygous genotype (two NHEJ-mediated mutations) was expected, a homozygous genotype was also expected where one mutated allele can serve as an HDR donor template to repair the second allele (HDR) or where micro-homology loci upstream and downstream of the cut 
A

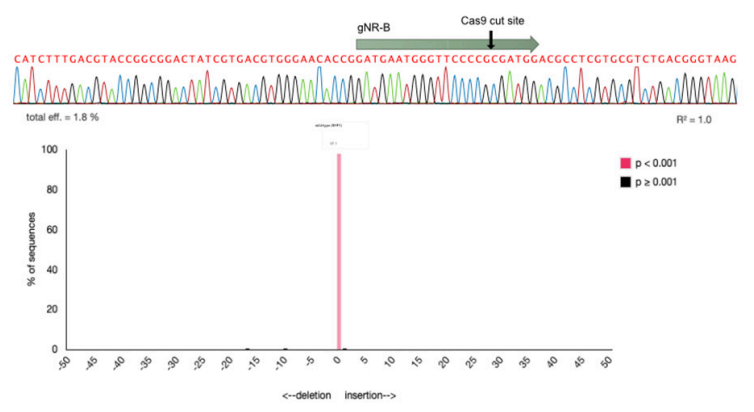

B

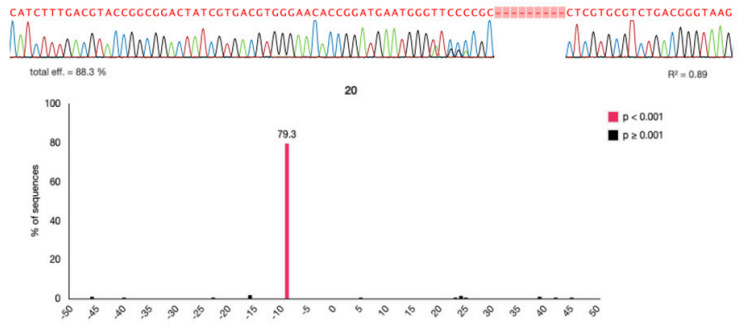

«-Medecion insertion $\rightarrow$
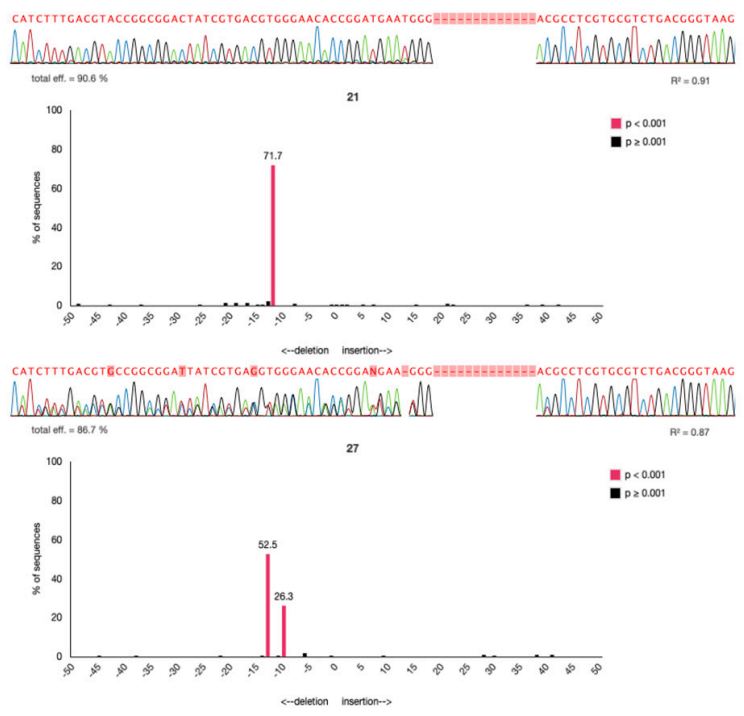

C

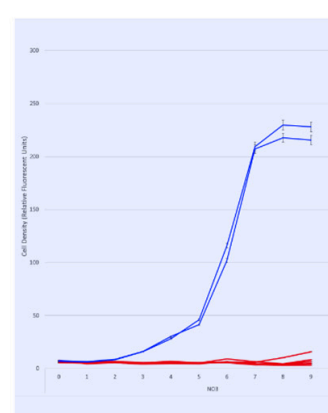

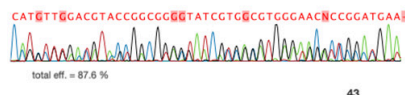
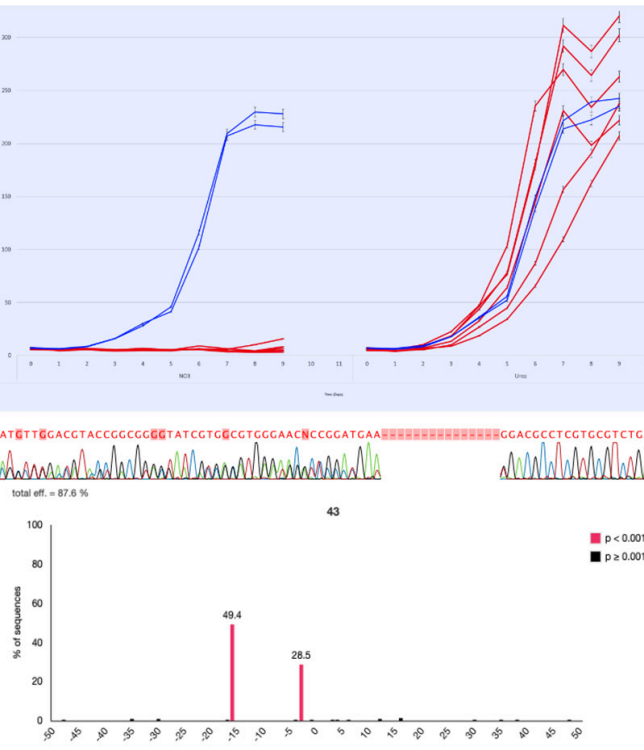

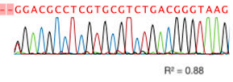

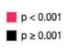

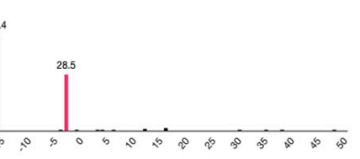

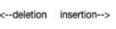
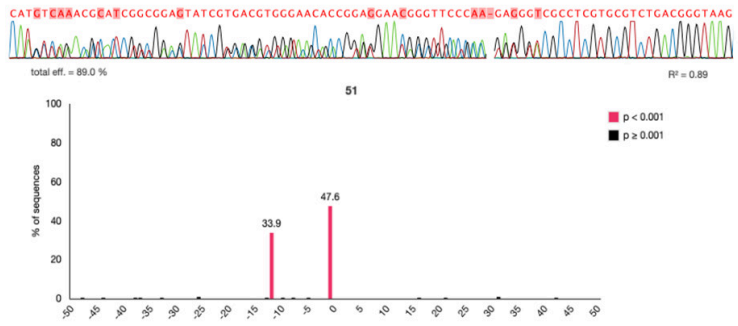

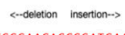
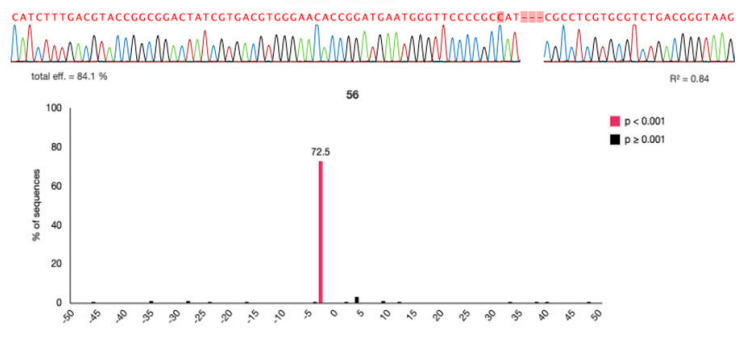

FIGURE 3 | Genotyping NR-KO mutant cell lines by colony sequencing and TIDE sequence analysis. (A) The sequencing alignment paired with a TIDE chart associated with a wild-type Phaeodactylum NR sequence. The gNR-B spacer and PAM are represented by the gray arrow and the Cas9 cut site by the black arrow. Below is the sequencing read, the associated electropherogram, and the TIDE result chart. The TIDE chart is a bar plot where the $x$-axis represents the predicted indel mutation ( 0 , wild-type, negative values, nucleotide deletion; positive values, nucleotide insertion) and the $y$-axis represents the percentage the specific mutation is of the entire sequence. A bar shaded pink indicates a $p$-value $<0.001$ for that predicted indel. Here, there is one pink bar at position " 0 " (wild-type) that represents $97.1 \%$ of the entire sequence (labeled above the bar). (B) Six NR-KO mutant cell line genotypes were assessed the same as wild-type. The wild-type sequencing read was used as a reference input for TIDE analysis to compare potential mutant sequence genotypes. NR-KO mutant genotyping was performed for all colonies using both manual sequencing curation and TIDE analysis (Supplementary Table S2) and candidate NR-KO cell lines were chosen for phenotype analysis. Here, six NR-KO candidates and their associated genotyping results are shown. Cell lines 20, 21, and 56 display a single peak in the negative values that indicates a homozygous deletion genotype. Cell lines 27, 43, and 51 display two peaks in the negative values that indicate two deletions or a heterozygous deletion genotype. (C) The six candidate NR-KO cell lines were validated to have lost nitrate reductase function when grown in liquid medium supplemented with nitrate. Compared to growth on urea (left panel), the NR-KO mutant could not grow on nitrate (right panel). Two wild-type cell lines are blue and the six NR-KO cell lines are in red. All line were grown in technical triplicates.

site anneal following strand re-sectioning, referred to as MMEJ (Microhomology-Mediated End Joining; Wang and Xu, 2017).

Since both hetero- and homozygous mutations were expected, the genotyping workflow was conceived such that resulting colonies could be screened following selection for Cas9-2A-sh ble expression (Figure 1C). Colonies were first picked and added directly to PCR mix to amplify the sgRNA expression cassette on the episome harbored within Phaeodactylum (Supplementary Figure S4). Only colonies that contained a correctly sized amplicon were chosen for genotyping. While 
Cas9 expression was not verified for each individual colony, a Cas9-Venus (E-YFP) fusion was visualized by confocal microscopy to ensure localization in the Phaeodactylum nucleus (Supplementary Figure S5).

The methods described above were used for the following two applications: single-gene mutagenesis and two-gene mutagenesis.

\section{Single Gene Mutagenesis}

Two sgRNAs were individually cloned into the Cas9-sh ble episome by golden-gate assembly (cloned episome product can be visualized in Supplementary Figure S2A). The sgRNAs were designed to mutate the nitrate reductase (NR) gene in Phaeodactylum. NR was picked as a genomic target because the function of NR has been well studied in Phaeodactylum and NR knockout cell mutants exhibit an easily screenable growth phenotype of cell death when supplemented with nitrate as a sole nitrogen source (McCarthy et al., 2017). The two sgRNAs were designed to target regions 55-bp apart so that the same amplification and sequencing primers could be used for genotyping efforts.

\section{Comparative sgRNA-Effectiveness Assessment of Cas9-Episome Transformed Phaeodactylum Population}

To quantify the mutagenesis efficiency for both sgRNAs population-wide, the T7 endonuclease assay was used (Figure 2). While the T7 assay has been typically used to genotype a clonal cell line, this method was useful to quantify the mutagenesis efficiency of all cells after transformation and phleomycinselection of Cas9-2A-sh ble. This was done with the intention to identify sgRNAs that may produce low rates of mutagenesis and subsequently discard them. The sgRNAs were designed to target the NR locus 55-bp apart (Figure 2A). This allowed both targeted populations (one for gNR-A and one for gNR-B) to be subjected to the same T7 assay parameters (Figure 2B). The T7 enzyme cleaves mismatched nucleotides and should cut at Cas 9 target loci with NHEJ-mediated mutations. The expected band sizes after T7 digestion was 127-bp and 58-bp for gNR-A and 113-bp and 72bp for gNR-B (Figure 2C). Homoduplexes, annealed bands that perfectly match during "heteroduplex" formation (Figure 2B), was expected to be observed in the T7 migration gel because biallelic knockouts that arise from MMEJ-mediated repair may, in theory, anneal to each other and avoid T7 cleavage. It was observed that gNR-A insufficiently targeted the NR locus $(0 \%$ mutagenesis efficiency) while gNR-B mutated the NR locus at $38 \%$ (Figure 2D).

By employing this method prior to colony genotyping, sgRNAs with low rates of mutagenesis throughout the population can be ignored which saves on reagent costs and labor. Henceforth, gNR-A was discarded from future targeting experiments due to its low mutagenesis efficiency compared to gNR-B.

\section{Picking and Genotyping Phaeodactylum_NR-KO Mutants}

TIDE (Tracking Indels by DEconvolution) sequence analysis was used to genotype Phaeodactylum cell lines (Figure 3). To do so, each sequence of the gNR-B target locus was first curated and analyzed manually. Next, one of two paired-end sequencing files were entered into the TIDE software and compared to a wild-type sequence for each colony. TIDE outputs a bar plot with predicted indel (insertion or deletion) mutations based on the inputted electropherogram compared to the wild-type. In Figure $\mathbf{3 A}$, a wild-type NR sequence read is shown above the TIDE result plot that compares wild-type to wild-type. The TIDE plot displays one bar at $x$-axis position "zero" and with a $y$-axis value of 97.1\% (displayed above bar). A pink colored bar also indicates the predicted indel was statistically significant $(p<0.001)$. This result indicates that there is a zero-nucleotide indel mutation at the predicted Cas 9 cut site that comprises $97.1 \%$ of the total sequencing reads. Clearly, the TIDE plot validates the sequencing read as a wild-type genotype.

Figure 3B shows six NR knockout mutant genotypes for cell lines 20, 21, 27, 43, 52, and 56. Cell line 20, for example, appears to have a bi-allelic mutation that is homozygous because the TIDE chart shows one pink peak that corresponds to a deletion mutation of 9-bp. Cell line 43, for example, appears to have a bi-allelic mutation that is heterozygous because the TIDE charts shows two pink peaks corresponding to a deletion genotype of 3-bp (28.5\%) and 15-bp (49.4\%). All six NR-KO genotypes display either homozygous or heterozygous genotypes by their TIDE charts. They also lack any peak at the "zero" position that would indicate that there was a wild-type contamination. Furthermore, cell lines with TIDE charts with only 1 or 2 indel peaks are shown here; additional peaks would indicate that the cell lines were not clonal and need to be re-streaked to find a clonal mutant.

Manual sequencing curation and TIDE analysis was performed for 80 gNR-A colonies and 64 gNR-B colonies. 2/80 (2.5\%) gNR-A colonies and 26/64 (40\%) gNR-B colonies contained a Cas9-mediated mutation. Here, the Cas9-sgRNA mutagenesis efficiencies of $0 \%$ and $28 \%$ calculated using T7 (Figure 2) reflects the efficiencies observed using TIDE.

Lastly, the nitrate growth assay was used to correlate the TIDE genotyping efforts to the expected NR mutant phenotype. The six mutant cell lines identified using TIDE did not grow on nitrate media compared to growth on ammonium media (Figure 3C).

\section{Two-Gene Mutagenesis}

The Cas9-2A-sh ble episome was assembled to harbor two sgRNA, one targeting glutamine synthetase 2 (GS-2, Gene ID: Phatr3_J51092) and one targeting a chloroplast-localized glutamate synthase (cGOGAT, Phatr3_J24739). The episome was assembled by golden-gate cloning and built to harbor both sgRNA expression cassette flanking a LacZ bacterial expression cassette (Figure 4A and Supplementary Figure S3).

\section{TIDE Genotyping of Phaeodactylum Two-Gene Mutant Cell Lines}

A total of 28 transformed Phaeodactylum colonies and 4 wild-type colonies were picked and genotyped using manual sequencing curation and TIDE sequencing analysis (Figure 4). The genotyping was performed successively. First, all 28 colonies were screened for mutation at the GS-2 target locus. Second, 
A

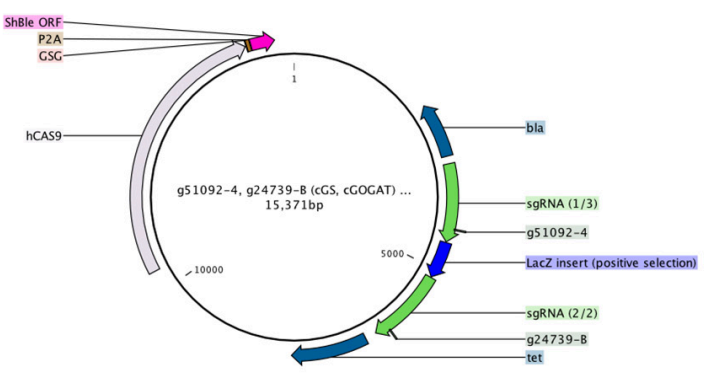

B

\begin{tabular}{|l|c|}
\hline Total cell lines screened & 32 \\
\hline Mutant cell lines & 28 \\
\hline Wild-type cell lines & 4 \\
\hline & \\
\hline Colonies w/ 51092 mutation & 6 \\
\hline 51092 mutants w/ 24739 mutation & 4 \\
\hline
\end{tabular}

C

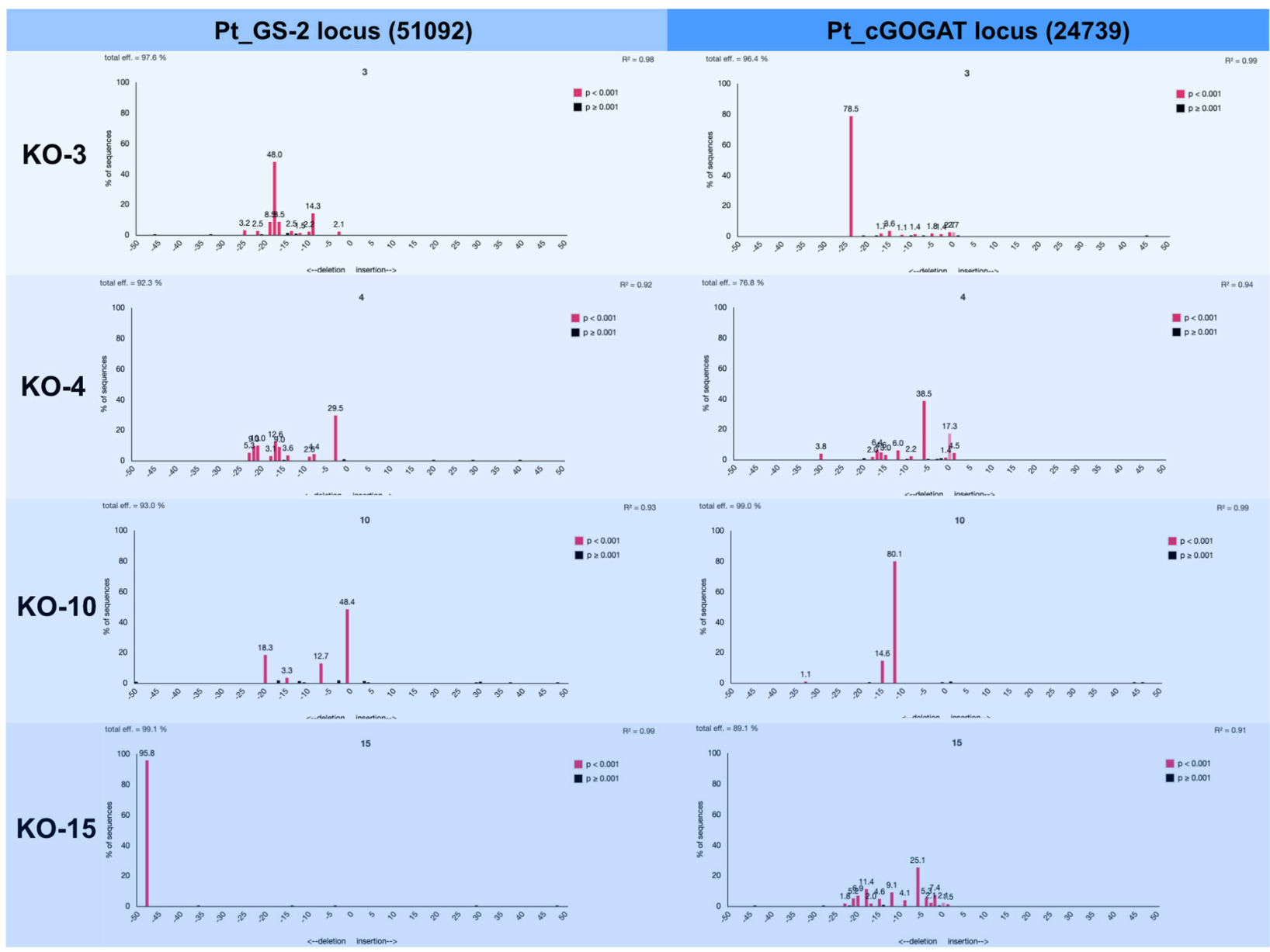

FIGURE 4 | Two-gene mutagenesis of the genomic target glutamine synthetase 2 (GS-2, Phatr3_J51092) and the chloroplast localized glutamate synthase (cGOGAT, Phatr3_J24739). (A) The assembly Cas9-sh ble:sgRNA episome plasmid map with two sgRNA expression cassette (g51092-4 and g24739-B, green arrows). (B) A total of 28 phleomycin-resistant colonies and 4 wild-type cell lines were genotyped for mutations at both target loci. The screening was performed iteratively where all 32 cell lines (mutants and wild-types) were first screened for mutation at the g51092-4 target locus then candidate GS-2-KO cell lines were screened for mutation at the g24739-B locus. 6/28 cell lines exhibited Cas9 activity in 51092 and 4/6 GS-2 mutants exhibited Cas9 activity in 24739 . The genotyping results for all cell lines was performed this way (Supplementary Tables S3, S4). (C) Four knock-out cell lines (3, 4, 10, and 15) and their associated TIDE charts show the diversity of mutagenesis at both loci.

GS-2 KO candidate colonies were screened for mutation at the cGOGAT target locus. Therefore, genotyping information is not provided for all 28 colonies for the cGOGAT target locus. The genotyping results using manual curation and TIDE analysis for each colony is provided in Supplementary Table S3 (GS-2) and Supplementary Table S4 (cGOGAT).
A total of $6 / 28(21 \%)$ colonies contained a Cas9-mediated mutation(s) at the GS-2 target locus and 4/6 GS-2 mutant candidates also contained Cas9-mediated mutations at the cGOGAT target locus (Figure 4B). Figure 4C shows the TIDE plots for each double-knock-out cell line $(3,4,10$, and 15) for both target loci (GS-2 on the left, cGOGAT on the right). 


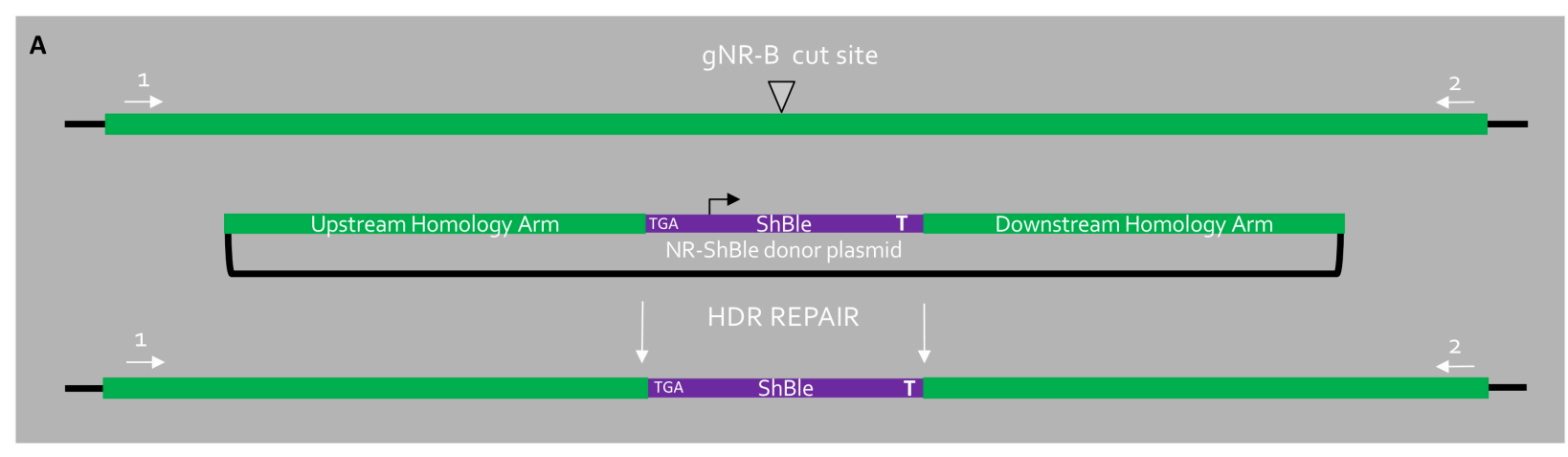

B

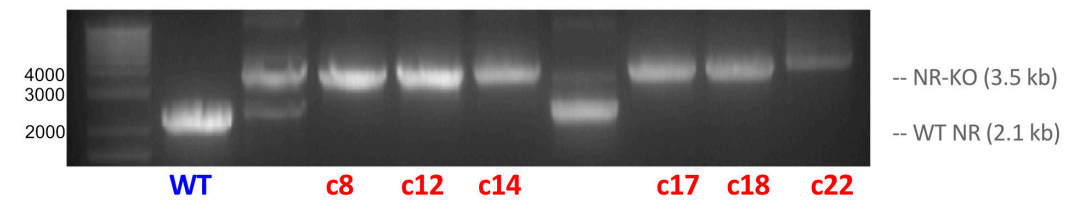

C
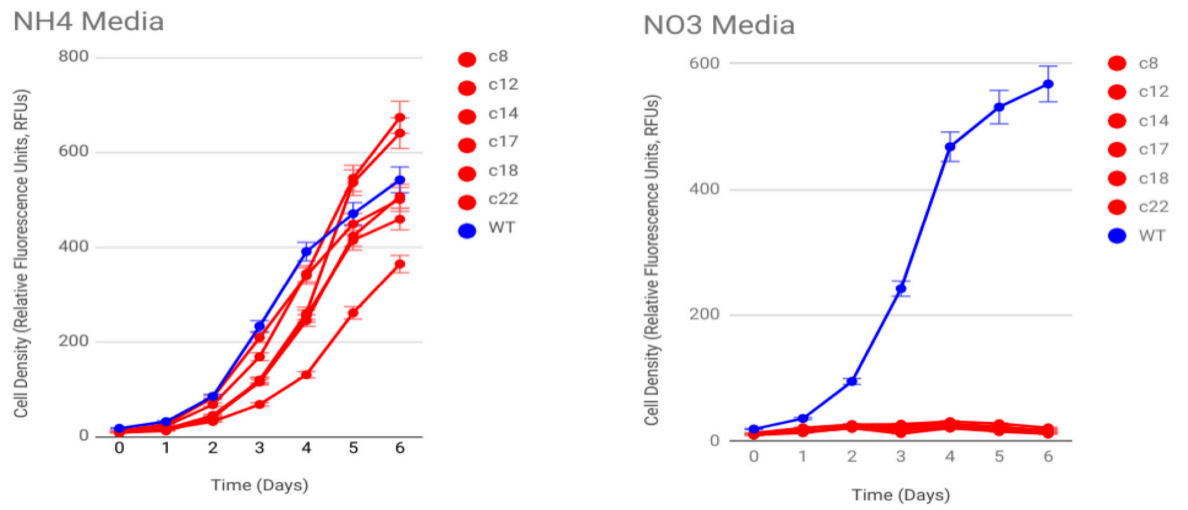

FIGURE 5 | HDR-mediated CRISPR-Cas9 targeted insertion of Sh ble gene via micro-particle bombardment genetic transformation. (A) An sh ble expression cassette with an upstream premature STOP codon was precisely inserted at the gNR-B DSB site Cas9, gNR-B, and a NR-Sh ble donor plasmid were delivered to P. tricornutum cells on separate plasmids by micro-particle bombardment. The plasmids are presumably integrated within genomic DNA and the CRISPR components are expressed from P. tricornutum chromosomes. (B) An electrophoresis gel shows migrated amplicon from six mutants target loci compared to a wild-type NR locus. A single band, rather than two or more, indicated a bi-allelic HDR-mediated edit of the gNR-B target. (C) All six mutant cell lines could not grow on nitrate-supplemented media compared to wild-type but could grow on ammonium-supplemented media, a confirmation the predicted NR-KO mutant phenotype. All cell lines were grown in technical triplicates.

Some mutations appear bi-allelic and homozygous, such as cell line 15 at the GS-2 locus (48-bp deletion) and cell line 3 at the cGOGAT locus (24-bp deletion). Only cell line 10 at locus cGOGAT displayed a bi-allelic, heterozygous mutation of a 15-bp deletion and 12-bp deletion. The remaining genotypes were not as clearly defined and have multiple (more than two) peaks in their TIDE chart and/or they contain a trace of a wild-type sequence (both examples are shown in cell line 4 at the cGOGAT locus).

\section{HDR-Mediated Gene Editing of NR in Phaeodactylum by Micro-Particle Bombardment}

Six cell lines were produced via HDR-mediated gene editing of the NR gene in Phaeodactylum (Figure 5). It should be noted that, although attempted, HDR-mediated mutagenesis was not observed when the HDR-donor was encoded on the Cas9-sh ble episome (results not shown). All six cell lines exhibited the genotype (Figure 5B) and phenotype (Figure 5C) of having an HDR-mediated insertion of the sh ble expression cassette at the gNR-B target locus. An efficiency of $17 \%$ for producing a bi-allelic, HDR-mediated Pt_NR mutant was achieved.

\section{DISCUSSION}

CRISPR-Cas9 mutagenesis was performed in the model diatom $P$. tricornutum specifically to streamline the use of bacterialconjugation transformation. First, the episomal delivery system for Cas9 was re-engineered so Cas9 could be selected for by phleomycin-antibiotic pressure. The $2 \mathrm{~A}$ peptide transcriptionally fused Cas9 and the selective gene, sh ble, to ensure Cas9 could be selected for after conjugation. Previous attempts to deliver Cas9 in an expression cassette separate from the sh ble cassette resulted 
in low mutagenesis efficiency when colonies were not initially screened for a predicted phenotype, such as NR phenotype. Although not reported here, previously published experiments that delivered a non-selectable Cas9 via bacterial conjugation in Phaeodactylum resulted in mutagenesis efficiencies ranging from 25 to $33 \%$ using one sgRNA target (Sharma et al., 2018). Here, mutagenesis efficiencies ranged from 2 to $40 \%$ when targeting the nitrate reductase gene. Despite reporting a higher mutagenesis efficiency when using gNR-B, mutagenesis efficiencies cannot be directly compared when targeting two different genomic loci.

It has been observed that only $10 \%$ of transformed colonies contained a full Cas 9 expression cassette, drastically lowering the mutagenesis efficiency (results not shown). It has been previously reported that the Phaeodactylum episome, naked, only retains its full plasmid sequence after transfer from $E$. coli to Phaeodactylum at 30\% (Karas et al., 2015; Diner et al., 2016). Such a Cas9 episome design would not be ideal for targets without a previously known phenotype. The $2 \mathrm{~A}$ peptide proved valuable in increasing the efficiency of Cas9 delivery to Phaeodactylum and in identifying mutant cell lines prior to checking for a phenotype response. Due to a transcriptional fusion to sh ble, it was presumed that the full Cas9 coding region was retained in all transformed Phaeodactylum transformants, though PCR amplification of the Cas9 ORF was not performed post-conjugation.

Although not thoroughly quantified, selection for Cas9 seems to alleviate previously reported problems of producing nonclonal Phaeodactylum that led to inevitable re-streaking, waiting for clonal cell lines to appear, and re-picking (Weyman et al., 2014). TIDE sequence analysis provided a predictive genotype for each cell line that can be interpreted as homozygous, heterozygous, or mixed genotype. For gNR-B, 26/64 cell lines exhibited Cas 9 activity while 6 exhibited either a homo- or heterozygous genotype by TIDE analysis (Figure $3 \mathrm{C}$ ), which can be interpreted to mean that those cell lines were clonal. However, 20/64 lines that passed the same efficiency criteria (in methods) contained more than 2 peaks in the TIDE chart or contained a trace wild-type sequence and therefore they were not considered clonal. Clonality was inherently tested for, also, when the NR-KO cell lines were subjected to a nitrate growth assay. If a wild-type cell contaminated the culture there would be growth on nitrate. Nevertheless, none of the six NR-KO cell lines grew on nitrate.

While TIDE sequence analysis is a useful tool to quickly screen raw sequencing reads for Cas9-induced indels. Here, TIDE was useful in identifying cell lines that contained targeted Cas9 activity and in inferring genotype, however, it is not recommended to use TIDE to confirm a cell lines genotype. Rather, the gold standard of sub-cloning the target locus into a TOPO vector followed by sequencing is recommended after TIDE analysis. It should be noted that TIDE analysis is complementary to CRISPR/Cas9 activity factor (CAF) analysis that was also designed to deconvolve diploid genotypes after CRISPR/Cas9 targeting (Stukenberg et al., 2018).

Re-streaking Phaeodactylum mutant is also recommended after TIDE analysis. The TIDE chart generated for gNR-B cell lines (Figure 3) suggest that the genotypes are either homozygous or heterozygous and that the cell lines are clonal.
Nevertheless, TIDE is not the gold-standard for genotyping and assessment of clonality. As shown in Figure 4, not all TIDE plots indicate clonality and therefore re-streaking would be necessary to isolate double-knock-out mutant cell lines. All supplemental tables outline the genotyping efforts performed in this study. The last column for each table indicates the predicted genotype of the colony and suggests whether or not streaking the culture is recommended.

Lastly, the new Cas9 episome design decreased the time to produce and validate mutant cell lines. The time to produce visible colonies was 10-14 days, genotyping was 3 days, and phenotype screening was 10 days after re-picking. In total, the production of 6 Phaeodactylum mutants for the NR gene took 3-4 weeks compared to 4-8 weeks when using particle bombardment (Sharma et al., 2018). In a rapidly developing research landscape, optimization of time to produce mutant cell lines should not be a time-limiting step when there are questions regarding biological processes, ecological relevance, or biotechnology utility is an important goal.

\section{DATA AVAILABILITY STATEMENT}

The datasets generated for this study can be found in the MM Frontiers 2-27-29 Data Availability Document https:/docs. google.com/spreadsheets/d/1snw_Jao9sdCDy2Y7cC3tP33cNzZ0 xTxOQTdHUEaARME/edit\#gid=2069237253. Plasmid designed and mentioned in this manuscript will be made publicly available at the plasmid repository addgene.com.

\section{AUTHOR CONTRIBUTIONS}

MM was responsible for all of the molecular cloning, genetic transformations, genotyping assays, and writing. VB helped in plasmid and episome design, specifically for the conjugation protocols and implementation of the $2 \mathrm{~A}$ peptide, and other intellectual contributions. PG performed the phenotype assays. MT designed golden gate primers for the episome. JM helped in daily tasks and manuscript writing. AA contributed to intellectual design and scope of the study. All authors contributed to the editing and feedback for this manuscript.

\section{FUNDING}

This study was supported by the Gordon and Betty Moore Foundation Grants GBMF3828 and GBMF5006 (AA), National Science Foundation Grants NSF-OCE-1756884 and NSF-MCB1818390 (AA), United States Department of Energy Genomic Science Program Grant DE-SC0018344 (AA), and National Aeronautics and Space Administration Grant 15-EXO15_2-0158.

\section{SUPPLEMENTARY MATERIAL}

The Supplementary Material for this article can be found online at: https://www.frontiersin.org/articles/10.3389/fmicb.2020. 00005/full\#supplementary-material 


\section{REFERENCES}

Bailleul, B., Rogato, A., de Martino, A., Coesel, S., Cardol, P., Bowler, C., et al., (2010). An atypical member of the light-harvesting complex stress-related protein family modulates diatom responses to light. Proc. Nat. Acad. Sci. 107, 18214-18219. doi: 10.1073/pnas.1007703107

Bielinski, V. A., Bolt, T. M., Dupont, C. L., and Weyman, P. D. (2017). Episomal tools for RNAi in the diatom Phaeodactylum tricornutum. PeerJ Preprints 5:e2907v1. doi: 10.7287/peerj/preprints.2907v1

Brinkman, E. K., Chen, T., Amendola, M., and Steensel, B. V. (2014). Easy quantification assessment of genome editing by sequence trace decomposition. Nucleic Acid Res. 42:e168. doi: 10.1093/nar/gku936

Cermeño, P., Lee, J.-B., Wyman, K., Schofield, O., Falkowski, P. G. (2011). Competitive dynamics in two species of marine phytoplankton under nonequilibrium conditions. Mar. Ecol. Prog. Ser. 429, 19-28. doi: 10.3354/ meps09088

Cho, S. W., Kim, S., Kim, J. M., and Kim, J. S. (2013). Targeted genome engineering in human cells with the Cas9 RNA-guided endonuclease. Nat. Biotechnol. 31, 230-232. doi: 10.1038/nbt.2507

Cong, L., Ran, F., Cox, D., Lin, S., Barretto, R., Habib, N., et al. (2013). Multiplex genome engineering using CRISPR/Cas systems. Science 339, 819-823. doi: 10.1126/science. 1231143

Davis, A., and Chen, D. (2013). DNA double strand break repair via nonhomologous end-joining. Transl. Cancer Res. 2, 130-143.

Deltcheva, E., Chylinski, K., Sharma, C., Gonzales, K., Chao, Y., Pirzada, Z., et al. (2011). CRISPR RNA maturation by trans-encoded small RNA and host factor RNase III. Nature 471, 602-607. doi: 10.1038/nature09886

Diner, R. E., Bielinski, V., DuPont, C., Allen, A. E., and Weyman, P. (2016). Refinement of the diatom episome maintenance sequence and improvement of conjugation-based DNA delivery methods. Front. Bioeng. Biotechnol. 4:65. doi: $10.3389 /$ fbioe. 2016.00065

Falciatore, A., Casotti, R., LeBlanc, C., Abrescia, C., and Bowler, C. (1999). Transformation of nonselectable reporter genes in marine diatoms. Mar. Biotechnol. 1, 239-251. doi: 10.1007/pl00011773

Gao, C. (2018). The future of CRISPR technologies in agriculture. Nat. Rev. Mol. Cell Bio. 19, 275-276. doi: 10.1038/nrm.2018.2

Gibson, D. G., Young, L., Chuang, R. Y., Venter, J. C., Hutchison, C. A. I. I. I., and Smith, H. O. (2009). Enzymatic assembly of DNA molecules up to several hundred kilobases. Nat. Methods 6, 343-345. doi: 10.1038/nmeth.1318

Guschin, D. Y., Waite, A. J., Katibah, G. E., Miller, J. C., Holmes, M. C., Rebar, E. J., et al. (2010). A rapid and general assay for monitoring endogenous gene modification. Methods Mol. Biol. 649, 247-256. doi: 10.1007/978-1-60761-7532_15

Hopes, A., Nekrasov, V., Kamoun, S., and Mock, T. (2016). Editing of the urease gene by CRISPR-Cas in the diatom Thalassiosira pseudonana. Plant Methods 12:49.

Jinek, M., Chylinski, K., Fonfara, I., Hauer, M., Doudna, J. A., and Charpentier, E. (2012). A programmable dual-RNA-Guided DNA endonuclease in adaptive bacterial immunity. Science 337, 816-821. doi: 10.1126/science.1225829

Jinek, M., East, A., Cheng, A., Lin, S., Ma, E., and Doudna, J. (2013). RNAprogrammed genome editing in human cells. eLife 2:e00471. doi: 10.7554/eLife. 00471

Karas, B. J., Diner, R. E., Lefebvre, S. C., McQuaid, J., Phillips, A. P., Noddings, C., et al. (2015). Designer diatom episomes delivered by bacterial conjugation. Nat. Commun. 21:6925. doi: 10.1038/ncomms7925

Kim, J. H., Lee, S. R., Li, L. H., Park, H. J., Park, J. H., Lee, K. Y., et al. (2011). High cleavage efficiency of a 2A peptide derived from Porcine Teschovirus-1 in human cell lines, Zebrafish and Mice. PLoS One 6:e18556. doi: 10.1371/journal. pone. 0018556

LaFave, M., and Sekelsky, J. (2009). Mitotic recombination: why? when? how? where?. PLoS Genet. 5:e1000411. doi: 10.1371/journal.pgen.1000411

Mali, P., Esvelt, K., and Church, G. (2013a). Cas9 as a versatile tool for engineering biology. Nat. Methods 10, 957-963. doi: 10.1038/nmeth.2649

Mali, P., Yang, L., Esvelt, K., Aach, J., Guell, M., DiCarlo, J., et al. (2013b). RNAguided human genome engineering via Cas9. Science 339, 823-826. doi: 10. $1126 /$ science. 1232033

McCarthy, J. K., Smith, S. R., McCrow, J. P., Tan, M., Zheng, H., Beeri, K., et al. (2017). Nitrate reductase knockout uncouples nitrate transport from nitrate assimilation and drives repartitioning of carbon flux in model pennate diatom. Plant Cell 29, 2047-2070. doi: 10.1105/tpc.16.00910

McGinn, J., and Marraffini, L. A. (2018). Molecular mechanisms of CRISPR-Cas spacer acquisition. Nat. Rev. Microbiol. 17, 7-12. doi: 10.1038/s41579-0180071-7

Nymark, M., Sharma, A. K., Sparstad, T., Bones, A., and Winge, P. (2016). A CRISPR/Cas9 system adapted for gene editing in marine algae. Sci. Rep. 6:24951. doi: $10.1038 /$ srep24951

Pulz, O., and Gross, W. (2004). Valuable products from biotechnology of microalgae. Appl. Microbiol. Biotechnol. 65, 635-648. doi: 10.1007/s00253-004$1647-\mathrm{x}$

Ran, F. A., Hsu, P. D., Wright, J., Agarwala, V., Scott, D. A., and Zhang, F. (2013). Genome Engineering using the CRISPR-Cas 9system. Nat. Protoc. 8, 2281-2308. doi: 10.1038/nprot.2013.143

Rastogi, A., Murik, O., Bowler, C., and Tirichine, L. (2016). PhytoCRISP-Ex: a web-based and stand-alone application to find specific target sequences for CRISPR/CAS editing. BMC Bioinform. 17:261. doi: 10.1186/s12859-016-1143-1

Rogato, A., Richard, H., Sarazin, A., Voss, B., Cheminant Navarro, S., Champeimont, R., et al. (2014). The diversity of small non-coding RNAs in the diatom Phaeodactylum tricornutum. BMC Genom. 15:698. doi: 10.1186/14712164-15-698

Sambrook, J. F., and Russell, D. W. (eds) (2001). Molecular Cloning: A Laboratory Edition. New York, NY, NY: Cold Spring Harbor Laboratory Press.

Sanders, J., and Joung, J. (2014). CRISPR-Cas systems for editing, regulating and targeting genomes. Nat. Biotechnol. 32, 347-355. doi: 10.1038/nbt.2842

Serif, M., Dubois, G., Finoux, A. L., Teste, M. A., Jallet, D., and Daboussi, F. (2018). One-step generation of multiple gene knock-outs in the diatom Phaeodactylum tricornutum by DNA-free genome editing. Nat. Comm. 9:3924. doi: 10.1038/ s41467-018-06378-9

Sharma, A. K., Nymark, M., Sparstad, T., Bones, A. M., and Winge, P. (2018). Transgene-free genome editing in marine algae by bacterial conjugation comparison with biolistics CRISPR/Cas9 transformation. Sci. Rep. 8:14401. doi: 10.1038/s41598-018-32342-0

Slattery, S., Diamond, A., Wang, H., Therrian, J. A., Lant, J. T., Jazey, T., et al. (2018). An expanded plasmid-based genetic toolbox enables Cas9 genome editing and stable maintenance of synthetic pathways in Phaeodactylum tricornutum. ACS Syn. Biol. 7, 328-338. doi: 10.1021/acssynbio.7b00191

Stukenberg, D., Zauner, S., Dell'Aquila, G., and Maier, U. G. (2018). Optimizing CRISPR/Cas9 for the diatom Phaeodactylum tricornutum. Front. Plant Sci. 9:740. doi: 10.3389/fpls.2018.00740

Vinayak, V., Manoylov, K., Gateau, H., Blanckhaert, V., Herault, J., Pencreac'h, G., et al. (2015). Diatom milking: a review and new approaches. Mar. Drugs 13, 2629-2665. doi: 10.3390/md13052629

Wang, H., La Russa, M., and Qi, L. S. (2016). CRISPR/Cas9 in genome editing and beyond. Annu. Rev. Biochem. 85, 227-264. doi: 10.1146/annurev-biochem06015-014607

Wang, H., and Xu, X. (2017). Microhomology-mediated end joining: new players join the team. Cell Biosci. 7:6. doi: 10.1186/s13578-017-0136-8

Weyman, P., Beeri, K., Lefebvre, S., Rivera, J., McCarthy, J., Heuberger, A., et al. (2014). Inactivation of Phaeodactylum tricornutum urease gene using transcription activator-like effector nuclease-based targeted mutagenesis. Plant Biotechnol. 13, 460-470. doi: 10.1111/pbi.12254

Zhou, Y., Zhu, S., Cai, C., Yuan, P., Li, C., and Wei, W. (2014). High-throughput screening of a CRISPR/Cas9 library for functional genomics in human cells. Nature 509, 487-491. doi: 10.1038/nature1 3166

Conflict of Interest: The authors declare that the research was conducted in the absence of any commercial or financial relationships that could be construed as a potential conflict of interest.

Copyright $\odot 2020$ Moosburner, Gholami, McCarthy, Tan, Bielinski and Allen. This is an open-access article distributed under the terms of the Creative Commons Attribution License (CC BY). The use, distribution or reproduction in other forums is permitted, provided the original author(s) and the copyright owner(s) are credited and that the original publication in this journal is cited, in accordance with accepted academic practice. No use, distribution or reproduction is permitted which does not comply with these terms. 